\title{
La dynamique de changement organisationnel une théorie conversation/texte de la communication et ses implications
}

James R. Taylor

\section{CpenEdition}

\section{Journals}

Édition électronique

URL : http://journals.openedition.org/communicationorganisation/1619

DOI : 10.4000/communicationorganisation.1619

ISSN : 1775-3546

Éditeur

Presses universitaires de Bordeaux

Édition imprimée

Date de publication : 1 mai 1993

ISSN : 1168-5549

Référence électronique

James R. Taylor, « La dynamique de changement organisationnel une théorie conversation/texte de la communication et ses implications », Communication et organisation [En ligne], 3 | 1993, mis en ligne le 26 mars 2012, consulté le 10 décembre 2020. URL : http://journals.openedition.org/

communicationorganisation/1619; DOI : https://doi.org/10.4000/communicationorganisation.1619

Ce document a été généré automatiquement le 10 décembre 2020.

(C) Presses universitaires de Bordeaux 


\title{
La dynamique de changement organisationnel une théorie conversation/texte de la communication et ses implications
}

\author{
James R. Taylor
}

\section{Introduction}

1 Les théories de la communication élaborées dans la littérature classique sont typiquement fondées sur une notion de transmission de messages dans des réseaux. Elles sont donc statiques dans leur application: elles expliquent bien le cheminement des messages dans des réseaux déjà établis, mais plus difficilement l'émergence et l'évolution de ces mêmes réseaux. Elles font ainsi abstraction de la dimension organisationnelle en tant que telle. Dans un contexte de transformation mondiale des entreprises publiques et privées, une telle restriction n'est plus admissible. Le défi actuel du manager n'est pas simplement de faire mieux fonctionner le dispositif organisationnel dont il ou elle a hérité par des améliorations ponctuelles des modes de communication au sein de l'entreprise existante mais davantage d'envisager de nouveaux modèles de gestion, plus adaptés à un environnement qui devient quotidiennement plus turbulent et imprévisible (Taylor \& Van Every, 1993).

2 Dans cet essai nous nous proposons d'esquisser, dans ses grandes lignes, une théorie de la communication pouvant sous-tendre une approche au changement organisationnel. Cette théorie est inspirée par une réinterprétation de la notion de réseau afin d'incorporer une dimension transactionnelle à côté de celle de l'interaction. Cette théorie qui oppose les modes de communication conversationnel et textuel, est le résultat d'une étude en communication organisationnelle que nous explorons à l'Université de Montréal depuis déjà quinze ans (Akzam, 1992 ; Cooren, inédit ; Giroux \& Taylor, 1988, en préparation ; 
Gurd, en préparation ; Hovey, 1992 ; gourdonnais, 1992 ; Lerner, en préparation ; Saumier, en préparation ; Saumier \& Taylor, 1990 ; Robichaud, 1991 ; Taylor, 1978a, 1978b, 1982a, 1982b, 1986, 1988,1990, 1991, 1992, 1993, soumis; Taylor \& Dyke, inédit; Taylor \& Robichaud, 1992 ; Taylor \& Van Every, 1993, en préparation).

\section{L'organisation en tant que communication}

3 Le concept d'organisation est une abstraction. Une organisation n'appartient pas au monde du matériel puisqu'elle ne correspond pas au domaine des objets que nous pouvons toucher, voir, entendre, flairer, goûter ou, d'une façon générale, percevoir par les sens. Elle appartient à l'univers des objets imaginés. Un objet imaginé n'est pas nécessairement moins réel qu'un objet physiquement présent à nos sens (Whitehead, 1925) mais son appréhension présente à la fois un problème d'ontologie et d'épistémologie: le problème n'est pas seulement de pouvoir le visualiser (conceptualiser) mais aussi de pouvoir vérifier, par des évidences concrètes les propriétés d'un objet qui ne doit son existence qu'à l'imagination.

4 Comment, en particulier, une entité qui appartient à la sphère de l'abstrait peut-elle « changer » ou " évoluer » ou "s'adapter » ou «se transformer »? Lorsqu'on parle, par exemple, de «changement de culture » ou «changement de structure d'entreprise » ou «implantation d'un programme de qualité totale» de quoi parle-t-on? S'agit-il d'une abstraction sans réfèrent, d'un contenant sans contenu, ou de quelque chose de réel ? Jusqu'à maintenant les sciences de la communication n'ont pu répondre à ce genre de question.

5 Une des stratégies habituelles des chercheurs en organisation et des praticiens confrontés à une telle interrogation est d'exploiter une métaphore (Morgan, 1989) : l'organisation devient, selon l'école, un organisme, une machine, un cerveau, etc. Le concept de changement prend alors un sens dicté par le caractère de la métaphore préférée, par exemple, l'adaptation (si on conçoit l'organisation comme une forme d'organisme) ou le redésigna (si l'on croit qu'elle est une machine).

6 L'avantage d'une métaphore est qu'elle illumine des facettes de l'expérience qui sinon resteraient obscures, mais le risque, puisque toute métaphore est, par définition, une représentation partielle et imparfaite de l'objet visé (Morgan, 1989), est de prendre la métaphore trop littéralement et ainsi de prêter à l'organisation des qualités qu'elle ne revêt pas en réalité, et d'en cacher d'autres qu'elle possède véritablement.

7 C'est aussi se doter d'une base fragile pour la gestion et la planification car, dans le quotidien, c'est souvent l'abstraction imaginaire qui évolue - et non pas la réalité ! La fiction peut facilement se substituer aux faits.

Notre manière de procéder est différente.

9 Nous partons du constat simple que toute organisation est médiatisée par la communication. En l'absence de communication, aucune organisation humaine ne pourrait continuer à exister, ne serait-ce qu'une fraction de seconde. Une organisation est à toutes fins pratiques un artefact de la communication. La solution au dilemme de sa conceptualisation (et ainsi la conceptualisation de son changement) est de découvrir et d'élaborer précisément les caractéristiques de la communication qui mettent en évidence les propriétés typiques de l'expérience organisationnelle. 


\section{L'importance de la conversation}

10 La manifestation habituelle de la communication est la conversation. Bien que la médiatisation des échanges humains par la technologie est omniprésente dans la société contemporaine, il demeure néanmoins vrai que, tout comme Mintzberg (1973) et d'autres observateurs des pratiques managerielles ont pu le constater (Weinshall, 1979), la plupart des activités de gestion prennent la forme d'une conversation en face-à-face. Donc il s'ensuit, si nous tenons à respecter nos engagements ontologiques, que le changement organisationnel équivaut à une transformation de sa conversation. Puisque le modèle traditionnel de réseau escamote la dimension conversationnelle le premier défi de la théorisation est de concevoir la conversation de manière à capter le phénomène organisationnel.

11 Précisons ici que notre objectif n'est pas de présenter un modèle de la conversation en tant que tel (il y a déjà une vaste littérature qui porte là-dessus, littérature que nous n'avons aucunement l'intention de résumer ou d'analyser), mais plutôt un modèle de l'organisation, compte tenu de la présupposition que l'organisation se manifeste à travers la conversation. En d'autres mots, nous sommes à la recherche des propriétés de la conversation qui sont révélatrices de la réalité virtuelle plus profonde derrière la surface de la parole. C'est cette réalité inéluctablement virtuelle qui est l'organisation, créée et soutenue par la communication - une organizing (Weick, 1979) parce qu'elle ne peut jamais être totalement réalisée, encore moins concrétisée (même si elle génère régulièrement des artefacts matériels par ses productions).

12 L'organisation, selon nous, apparaît dans la conversation (et n'est pas que véhiculée par la conversation) (Dewey, 1916).

\section{L'importance du texte}

13 Si l'organisation se révèle dans la conversation, elle ne devient reconnaissable que lorsqu'elle est manifestée dans une forme qui convient aux procédés du fonctionnement cognitif. Elle doit être perçue avant d'être reconnue. Elle doit devenir un objet de discours avant d'être un objet de gestion et de planification.

14 Cette traduction d'interaction en cognition est pour nous l'amorce de la démarche de la textualisation. Le propre d'un texte est d'appartenir à la sphère du lisible ou de l'interprétable. Une organisation qui n'est pas textualisable, n'est pas reconnaissable. C'est par le biais de sa textualisation que l'organisation reçoit le statut d'objet et devient ainsi quelque chose de manipulable - et de changeable. L'organisation en tant qu' intersubjectivité manifestée en interaction demeure inévitablement virtuelle, comme nous l'avons dit. C'est dans sa textualisation qu'elle acquiert un sens. L'objet organisation n'est ni une machine, ni un organisme, ni un programme, ni un cerveau - mais plutôt un texte. C'est cette distanciation produite par le texte (Ricœur, 1971, 1975,1986) qui nous permet d'en parler, et d'agir « rationnellement » en conséquence. Le rapport texte/conversation est fondamental pour une compréhension du phénomène de changement. Ce rapport est médiatisé par la transaction. 


\section{La transaction : La charnière entre la conversation et sa textualisation} ensemble de transactions constituent une organisation : l'unité de transaction est à la fois le principe d'articulation de la conversation et du texte, ainsi que l'élément de base de leurs structurations respectives. Les transactions se réalisent en conversation par le truchement de l'interaction ; les transactions sont également la matière d'une perception cognitive et d'une éventuelle représentation textuelle.

\section{Prémisse}

Une organisation est un ensemble de transactions. Une transaction est une unité de communication qui comporte un échange de valeurs d'une personne à une autre; toute communication est un système de création et de transmission de valeurs (et non pas simplement de messages).

L'image conventionnelle de l'organisation que l'on retrouve dans la plupart des textes portant sur la communication est celle d'un réseau. Dans cette façon de voir, les parties de l'organisation sont des individus (ou leurs simulacres) qui reçoivent et envoient des messages (selon la définition de leur tâche). Ils figurent dans le réseau comme des noyaux de traitement d'information. Les liens de transmission qui relient les individus forment les interconnections du réseau. Le réseau est l'organisation.

Un organigramme est l'exemple type du réseau, ainsi conçu.

Nous proposons une approche différente :

Pour nous, ni l'existence des individus, ni même leur composition en réseaux par la transmission de messages, ne sont en soi des garanties de l'existence organisationnelle. Une organisation existe à partir du moment où il y a reconnaissance des engagements mutuels. Etre organisé veut dire être en relation. C'est la relation qui est la matière de l'organisation. Le « réseau » qui nous intéresse est donc un agglomérat de relations.

Nous ne nions pas la validité de la théorie conventionnelle en tant que description d'un aspect particulier de la communication, soit la transmission de signaux interprétables comme des messages, mais cette théorie nous semble trop limitée dans son applicabilité car elle n'explique ni la structuration du travail ni la sociabilité des membres. Elle illustre le phénomène superficiel de l'échange de messages mais elle ne nous montre nullement le processus d'organisation en tant que tel.

23 Au fond, notre difficulté avec le modèle « réseau » est celle-ci parce qu'il fournit une carte de l'organisation (le concept de réseau provient d'un champ des mathématiques: la « topographie ») il présume que l'organisation est déjà établie; il prend pour acquis que le territoire a déjà été exploré, ce que nous nions car, pour nous, le territoire -lorsque l'objet est communicationnel - est une création de la carte qu'elle prétend montrer autant que le contraire. Il y a alors ce que Leshan \& Moreau (1982) appellent feed-back épistémique parce que, comme nous allons tenter de montrer, l'organisation reflète nos idées autant que nos idées reflètent l'organisation.

Communication et organisation, 3 | 2012 
24 Notre approche est empirique ; elle présume, au contraire, que le défi est toujours de dresser le plan de l'organisation. Ce qu'il nous faut trouver d'abord ce sont des instruments qui nous permettent de cadastrer adéquatement la commune. Notre unité de mesure, dans cette entreprise, est la relation véhiculée par des transactions.

La réalisation des relations (concept statique) suppose l'achèvement des transactions (concept dynamique). La relation s'établit par un rapport de valeurs transmises. D'après Salancik \& Leblebici $(1988$, p. 7) une transaction est « un accord entre des parties qui détermine ce que chacun d'eux fera afin de créer une valeur par leur relation " ( a transaction is an arrangement between parties about what each of them shall do to realize value through their relationship »). Une transaction incorpore un échange, mais le concept de transaction englobe celui d'échange car il spécifie à la fois les partenaires à l'échange, les biens à échanger et les droits et obligations de chaque partie à la transaction, futurs et passés. La description appropriée d'une organisation n'est plus celle d'un organigramme décrivant un ensemble de postes, chacun avec ses devoirs et pouvoirs, mais plutôt celle d'un ensemble de transactions, avec lesquelles on associe des «transigeants ». La modification de perspective est fondamentale lorsqu'on passe du statique au dynamique, du codifiable au négociable, de relativement fixe à en continuelle mutation.

\section{Prémisse}

Puisqu'une transaction est basée sur l'échange d'un objet de valeur, elle suppose comme condition antécédente, la création d'une valeur communicable.

Le concept de transaction, qui est au cœur de notre théorie, connote l'échange d'une chose de valeur d'une personne à une autre. La création d'une valeur suppose préalablement, l'existence d'un rapport sujet/objet, l'objet en question ayant dû subir une transformation d'un état initial (avant traitement) à un état final (après traitement). C'est précisément l'ensemble des transformations de ce genre qui justifie, économiquement, la place de l'organisation dans la société car ce sont les valeurs ainsi produites qui identifient son utilité sociale.

28 Le sujet (qui peut être un sujet collectif ou individuel) agit sur l'objet, à l'aide d'une instrumentation quelconque, et c'est conséquemment à son action que la valeur est créée. La transformation modifie l'objet d'un état sans valeur économique (que nous pouvons appeler « état naturel ») à un état ayant une valeur économique appréciable (que nous appellerons « état acculturé »). On retrouve ici la tension de base entre nature et culture. La production de valeur implique que l'objet devient socialement utile, une situation que nous pouvons représenter ainsi :

1. «Nature » dans le SENS d'une matière brute non-consommable, et non pas d'une écologie. La tendon entre nature et production cet inhérente à la notion d'activité utilitaire, quelle que tort la positon politique adoptée, car la connotation du mot « cultiver » cet de travailler (la terre) pour la rendre fertile, ou l'améliorer. 
Figure 1. La création d'un objet de valeur en tant qu'effet de « socialisation » des produits naturels par l'intervention d'une pratique culturellement validée

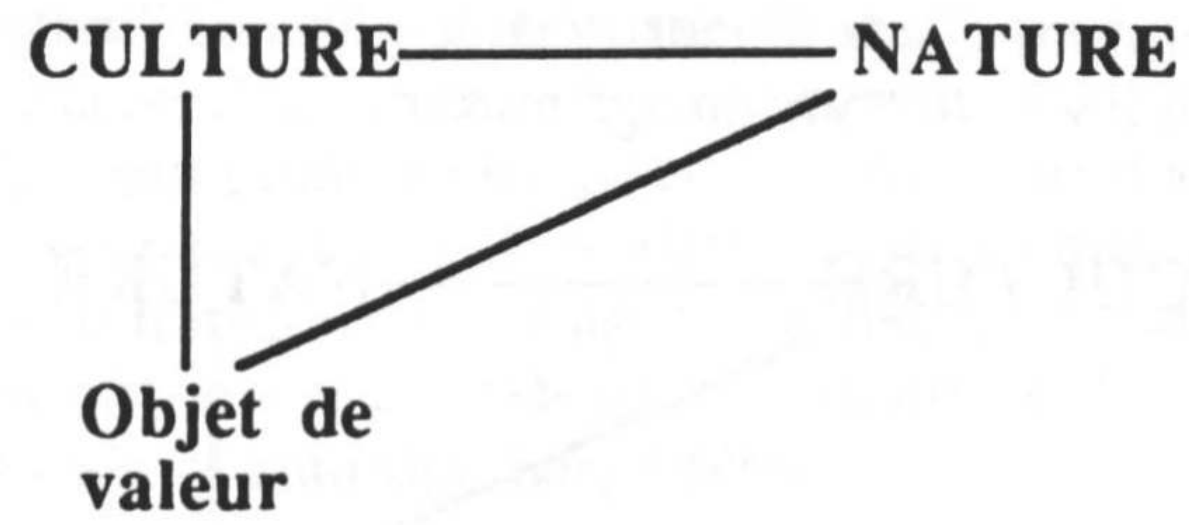

Le concept de valeur incorpore ainsi l'idée d'une opposition: un objet acquiert de la valeur non seulement parce qu'il est socialement utile mais aussi parce que sans sa transformation « culturelle » il n'aurait pas été naturellement consommable.

En linguistique (Halliday, 1970), l'objet sur lequel on opère s'appelle le patient et correspond, formellement, à l'objet direct d'un verbe transitif (ce dernier décrit alors l'opération). Le mot "patient» a une acception particulière dans ce contexte et ne correspond pas à l'emploi habituel du terme dans le langage du quotidien.

Le sujet, dans cette formulation, peut maintenant se transformer en agent car il se met dans une situation de communication habilitée : étant en possession d'un objet de valeur, il peut devenir agent pour quelqu'un à qui la valeur peut être transférée. (Sans transfert il n'y aurait pas eu de communication et, a fortiori, d'organisation.) Ainsi dans notre théorie la communication concerne toujours le transfert de valeurs. Le transfert de messages dont traite la théorie conventionnelle n'est pour nous qu'une manifestation superficielle d'un échange plus profond.

S'il y a des gains de valeur (valorisation d'un objet), il y a aussi des pertes de valeur (dévalorisation d'un objet) : la maladie, la pollution, la rouille ou toutes autres formes de détérioration d'une valeur déjà acquise. À l'instar de la figure 1, il y a alors une image miroir : 


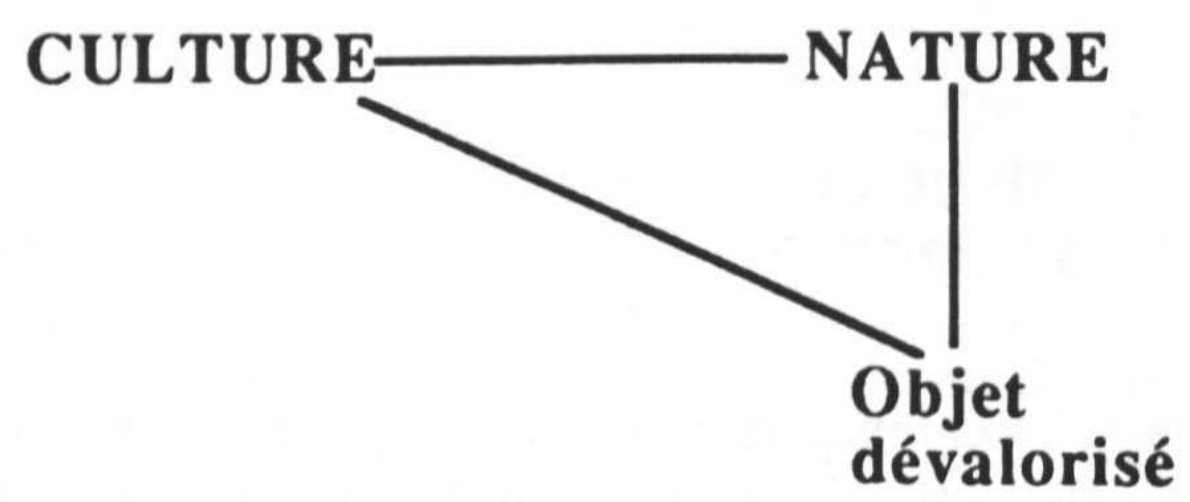

34 L'objet retourné à son état naturel (dévalorisé = déculturé) a ainsi perdu de valeur (ou a même acquis une valeur négative). Le retour de la tuberculose dans les sociétés occidentales dont parlent actuellement les experts en santé est ainsi à concevoir comme une revanche de la nature incontrôlée et une défaite pour la culture de la pratique médicale.

Si l'acteur peut se transformer en agent social c'est parce qu'il agit au nom de la société (comme un médecin, un policier, un cultivateur, par exemple). C'est cet investissement qui supporte la légitimité de l'organisation à laquelle il appartient. Mais, suivant la même logique, un anti agent (qui représente la nature incarnée sous la forme d'un agent opposé à la valeur culturelle, ou un anti-sujet) pourrait exister; non plus le Sida (une maladie) mais quelqu'un qui a, peut-être délibérément, infecté un ami avec la maladie (un malfaiteur). Il s'agit alors de délinquance et l'organisation associée, s'il y en a une, est illégitime. Donc les termes «culture » et "nature " peuvent être conçus soit comme des abstractions, soit comme des concrétisations, selon le contexte. Nous avons choisi les termes de «culture » et « nature » afin d'opposer des forces qui nous paraissent être à la base de la réalité organisationnelle, qui supposent toujours, selon notre façon de voir, une «amélioration» (Petit Larousse) de la situation naturelle afin de la rendre, au plan utilitaire, socialement consommable. L'organisation existe pour des buts instrumentaux socialement reconnus. Les abstractions «nature » et "culture » se transforment en agents (anti-agents) humainement identifiables, c'est-à-dire en sujets (anti-sujets), lorsqu'elles sont anthropomorphisées.

Inversement, par ailleurs, notre interprétation du rôle des agents identifiables, même dans le concret du quotidien, incorpore toujours une interprétation de leur investissement culturel/naturel. Le concept de valeur n'est jamais absent dans la communication humaine.

La transaction commence alors par la constitution d'une valeur qui détermine son investissement économique (pas nécessairement chiffré ou même chiffrable). Toute organisation incorpore un marché virtuel (voir à ce propos March \& Simon, 1958, et en particulier chapitre 4, ainsi que Chandler, 1977, Williamson, 1977, Clemons, 1989, Taylor \& Van Every, 1993). C'est par le biais de l'ensemble des transactions, réalisées dans l'interaction, que l'organisation se construit. C'est la configuration, ou le profil, des transactions primaires qui détermine le caractère de l'organisation: un vendeur de 
produits alimentaires se distingue d'un marchand d'automobiles, et se dernier joue un rôle distinct $\mathrm{du}$ fabriquant d'automobiles, qui est différent d'une entreprise de viticulture. C'est la nature des transactions qui prime dans la définition de la mission de l'entreprise.

\section{Le cas spécial de l'information}

38 La présentation jusqu'ici peut laisser l'impression que les transformations de base, qui aboutissent à la création d'une valeur et qui supportent ainsi la communication, sont uniquement d'ordre matériel, soit la production de biens ou de services. Notre intention est différente. Pour nous (Taylor 1988,1990), l'information est aussi le résultat d'une transformation de l'expérience brute (les données de la nature) en une interprétation culturellement fondée. Une information est autant un objet de valeur que tout autre don. L'administration est donc sujette à la même règle que les autres secteurs de l'entreprise : elle aussi est un système de transactions.

C'est un point auquel nous reviendrons dans notre discussion sur la conversation.

\section{Prémisse}

Le couplage transactionnel engendre la création de liens de réciprocité agent/ bénéficiaire (ou d'anti agent/victime). Le lien créé par la communication est à la fois économique et social.

41 Une transaction comporte l'échange d'un objet de valeur. Cette valeur est soit positive (dans le cas d'un don) ou négative (dans le cas d'un vol). La communication suppose le transfert d'une valeur d'une personne (la source) à une autre (le destinataire), cet échange étant médiatisé par une seconde relation qui réunit un agent (ou un anti agent) et un bénéficiaire (ou une victime). La source peut être agent ou bénéficiaire. Suivant le cas, le destinataire joue le rôle du complément. Le bénéficiaire est soit le patient de la transaction ou une partie intéressée dans le traitement du patient ; l'agent est soit luimême l'instrument de traitement ou son représentant. La détermination de l'identité du patient dépend de la valeur à échanger : source, si la valeur à échanger est négative (ex. une opération d'intervention chirurgicale afin d'enlever une tumeur), destinataire si la valeur à échanger est positive (ex. une opération de réaménagement d'un chalet à la campagne).

Il y a donc deux relations en cause, sujet/objet et sujet/sujet, cette dernière relation étant déterminée par l'orientation à l'objet: soit comme agent causal responsable de l'opération, soit comme la personne affectée par le résultat. Le tout forme un complexe triadique indissoluble.

43 La valeur est octroyée au bénéficiaire par l'agent dans le cas du don (ou arrachée de la victime par l'anti agent, dans le cas d'un vol) et doit être acceptée ou non (tolérée ou non, dans le cas d'un vol) par le bénéficiaire (ou la victime) pour que la transaction soit entérinée (ou non). Un don accepté signifie l'initiation d'un contrat réunissant agent et bénéficiaire. La communication a donc une base nécessairement contractuelle. C'est par les contrats que la permanence de la structure organisationnelle est assurée. 
qu'économique et utilitaire. La transaction est normalement réalisée pour le compte d'autrui (elle peut être commanditée ou volontaire), et le sujet-actant se transforme ainsi en agent, parce qu'il agit pour un bénéficiaire. Le rapport agent-bénéficiaire a donc un double caractère : il suppose le transfert de l'objet de valeur d'une source (ou destinateur) à un récepteur (ou destinataire) d'un côté, et simultanément de l'autre, l'établissement d'une obligation (ce que le Larousse appelle « un accord conclu sur la base de concessions réciproques ») du bénéficiaire à l'égard de l'agent. Cette obligation peut prendre la forme d'un paiement monétaire, peut également prendre la forme d'une rémunération éventuelle sous forme de service équivalent; la source est cette fois rendue par l'exbénéficiaire agissant maintenant comme agent pour l'ex-agent, qui devient ainsi bénéficiaire à son tour. Même dans une organisation à caractère bureaucratique (et nonmercantile), il subsiste une économie morale qui constitue la matrice organisationnelle (March \& Simon, 1958 ; Chandler, 1977 ; Williamson, 1977). Le principe s'applique même, paraît-il, à l'organisation des familles (Silverstone, 1990).

\section{Prémisse}

Il y a une équivoque qu'on ne peut supprimer dans la définition des rôles d'agent et de bénéficiaire car la différence entre les rapports agent/bénéficiaire et anti agent/-victime ne constitue qu'une inversion de perspective, sujette à interprétation. 

une seconde, celle de bénéficiaire/victime (Figure 3).

Figure 3. Le rapport agent/bénéficiaire dans son contexte d'oppositions logiques

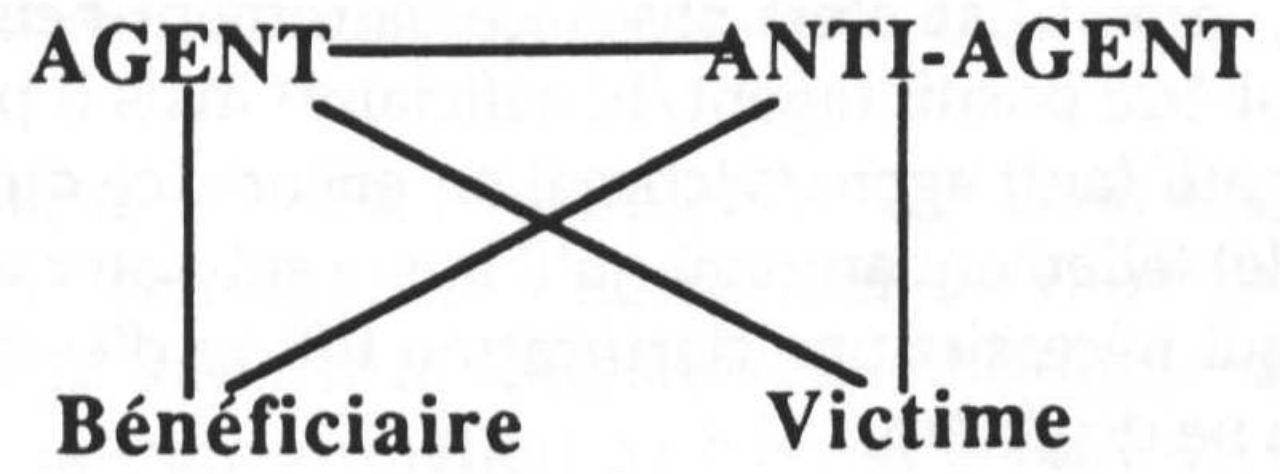
liens positifs (de type agent/bénéficiaire, selon notre interprétation). C'est là l'essentiel même de l'approche dite "fonctionaliste ». Tout conflit est traité comme une aberration regrettable de la normalité (une carence temporaire à corriger le plus rapidement possible). Mais malheureusement, la distinction entre agent/bénéficiaire et anti agent/ victime n'est souvent qu'une question d'interprétation. Les deux intervenants éventuels sont chacun des agents, l'ajout du préfixe « anti » n'étant qu'une attribution d'absence de sanction culturelle pour un acte donné. L'orientation du système d'oppositions (agent versus anti agent) dépend de la perspective adoptée par chaque personne, car ce qui est culturellement sanctionné ou non est une question de point de vue. Ce qui rend l'anti agent un «anti » c'est son opposition à l'agent mais la perspective est réversible : du point de vue de l'«anti » c'est l'agent qui est en opposition. Donc savoir si l'on est bénéficiaire ou victime est encore plus problématique car les deux agents se présentent normalement dans leur meilleures couleurs, chacun réclamant sa légitimité.

problème d'interprétation est ici général : le dilemme éternel de tout membre d'une organisation est de savoir si il est bénéficiaire ou victime lors d'une transaction où il se trouve engage, car la distinction dépend des propriétés de la situation et de l'interprétation des mobiles de l'agent avec qui l'on traite. Même si la transaction est réussie, le résultat n'est pas nécessairement heureux : le lien peut être positif (agent/ bénéficiaire) mais il peut aussi être négatif (anti agent/victime) ou encore (ce qui est plus probable) tellement ambigu qu'il laisse subsister une incertitude qui nécessite une clarification future d'éviter que la relation ne dégénère.

\section{Prémisse}

Malgré l'ambiguité, le rapport agent/bénéficiaire constitue le "matériau de construction" de l'organisation. Le concept de transaction incorpore deux principes de hiérarchisation. Par conséquent, les transactions s'arrangent naturellement en système. Le tout forme la structure, ou le squelette, de l'organisation. 

prise isolément. Nous allons maintenant montrer comment la transaction devient le matériau de construction de l'organisation. L'organisation en tant que telle est une configuration d'éléments, tout comme un squelette est compose d'os. La question se pose ainsi : quel est le principe d'articulation des composantes transactionnelles qui permet de former un tout structuré ?

complémentaires dans une chaine de production. Pour prendre un exemple concret, l'émission de télévision commence par une idée; l'idée doit être transformée en «traitement »; le traitement en script; le script doit être tourné ; le film qui en résulte doit être monté et le tout doit être réalisé afin de devenir émission ; finalement l'émission doit être promue, vendue et diffusée avant qu'elle puisse être "consommée " par le public. Cette logique de cheminement est dictée par la nature du matériel à traiter. C'est ce que Porter $(1980,1985)$ appelle une chaîne, ou un système, de valeurs. Compte tenu de ses enchâssements, la chaîne constitue une hiérarchie.

Prenons un cas. Un réalisateur de télévision fait appel à un service technique afin de monter un film qu'il veut incorporer dans son émission. Le montage du film constitue la transformation de base et établit un rapport entre un sujet (le monteur) et un objet (le film). Lorsque le montage est terminé, le film ainsi traité est acheminé au réalisateur, créant ainsi un rapport agent/bénéficiaire entre le monteur et le réalisateur (ce qui suppose une rémunération éventuelle du monteur, peut-être par une tierce partie, si le montage se fait au sein d'une organisation). Le réalisateur devient, à son tour, un producteur qui produit une émission pour un diffuseur, créant ainsi une autre relation agent/bénéficiaire. Il y a donc une suite de transactions séquentiellement couplées avant que soit atteint le public qui est le bénéficiaire final.

Figure 4

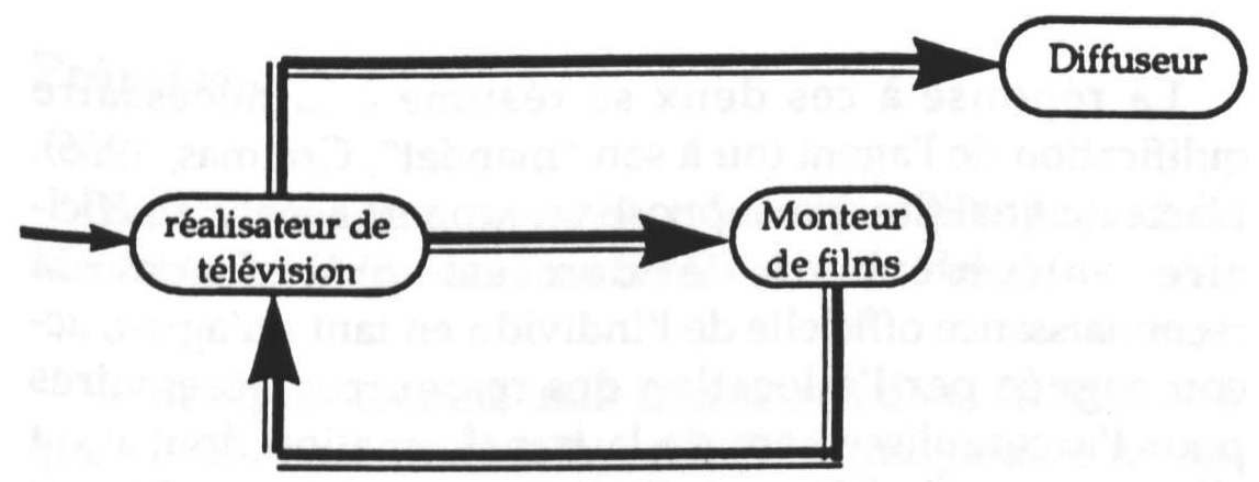

Le couplage de transactions dans un système de coordination (exemple suggéré par Mintzberg, 1979/1982)

À chaque stade, il y a une transformation qui crée simultanément des sujets (pour qu'ils puissent devenir subséquemment des agents) et des objets de valeur. Les transformations instaurent chaque fois une nouvelle situation de communication et un rapport agent/ bénéficiaire nouveau. La logique extrinsèque de la transformation matérielle impose une 
contrainte (un paramètre) sur la composition intrinsèque du système transactionnel. Nous voyons apparaître par conséquent la structure horizontale typique d'une organisation complexe.

Le principe de structuration verticale est différent. Le concept d'«agent» est fondamental à notre théorie. Mais d'où vient l'« agence » ainsi manifestée ? Comment estce qu'un sujet devient investi du statut d'agent?

Premièrement, puisqu'il s'agit d'une pratique culturelle acceptée, l'agent doit être socialement reconnu dans son rôle de transformateur d'objet, ou d'opérateur sur un patient. Une certaine autorité doit lui être accordée pour qu'il puisse jouer son rôle. D'où vient donc cette autorité ?

Deuxièmement, puisque l'intervention, dont il est chargé, suppose une instrumentation quelconque, éventuellement dispendieuse, il y a la question des ressources mises à la disposition de l'agent : d'où viennent-elles?

La réponse à ces deux se résume à la nécessaire qualification de l'agent (ou à son «mandat », Greimas, 1966). L'acte de qualification suppose un rapport agent/bénéficiaire antécédent où le don est précisément la reconnaissance officielle de l'individu en tant qu'agent, accompagnée par l'allocation des ressources nécessaires pour l'accomplissement de la transformation dont il ou elle sera chargée à l'avenir (la tâche qui lui est confiée, en d'autres mots).

63 L'agent a donc simultanément deux qualificatifs : au niveau du bénéficiaire, il est un donateur et au niveau de l'agent qui l'a qualifié, il est un bénéficiaire du privilège de jouer le rôle d'agent social et d'être reconnu comme ayant le droit d'agir ainsi. La récompense attendue par l'agent pour le bénéfice qu'il a conféré au bénéficiaire se réalise à un autre niveau, celui de sa qualification : elle n'est pas nécessairement directe. Le système ainsi produit est récursif. Le droit de donner à un bénéficiaire est alors le plus important bénéfice de tous, dans un contexte social : c'est par la possibilité d'être agent que la personne acquiert une identité et devient un membre de la société. M est littéralement, comme nous conseille la Bible, plus sanctifiant de donner que de recevoir.

64 L'ensemble des transactions menant à des qualifications d'agent répétées en cascade crée la hiérarchie organisationnelle. Le caractère spécifique de la forme verticale qui émerge $\mathrm{du}$ processus de qualification récursive est déterminé, pour chaque organisation particulière, par la distribution, et initiale et évolutive, des ressources, soit financières ou politiques, car c'est par le contrôle des ressources que le choix du bénéficiaire de la qualification est déterminé. Puisque la qualification constitue une autorisation d'utiliser les instruments de culture (y compris des droits limités de qualification des autres), la hiérarchie émergente est fondamentalement un système d'autorité et, comme nous allons maintenant le démontrer, d'identité.

Prémisse

\section{La transaction constitue le moyen par lequel les identités des individus sont créées et maintenues.}

66 L'idée que l'identité individuelle nait dans l'interaction, que l'expérience de la subjectivité est inséparable de son objectivisation n'est pas nouvelle: elle a été vigoureusement défendue par George Herbert Mead et a trouvé une expression plus récente dans les 
travaux de Erving Goffman, Herbert Garfinkel et des membres de l'école qu'il a inspirée (l'ethnométhodologie) ainsi que dans les travaux de l'école de Palo Alto, où l'on associe principalement les noms de Gregory Bateson et Don Jackson (Watzlawick et al, 1967). Pour Mead, par exemple, on devient un soi par un effet de réflexivité : le je et le moi naissent dans la même séquence. Des recherches interculturelles récentes (ex., Kondo, 1990) démontrent encore plus clairement que l'identité d'une personne se construit par, et à travers, l'interaction et que l'existence individuelle dépend des procédés de l'interaction sociale. Les identités des acteurs organisationnels sont tributaires de la définition des transactions qu'ils sont autorisés à entreprendre (ou qu'ils réussissent à s'approprier d'eux-mêmes).

Une condition de base pour la réussite d'un acte de communication, dans un contexte de transactions, est que la personne en question doit être la bonne personne, c'est-à-dire qu'elle doit être autorisée à entreprendre l'acte en question (Austin, 1960). Le corollaire de ce constat, en revanche, est que l'identité de la personne est déterminée par les transactions dans lesquelles elle s'engage (ou dans lesquelles elle est autorisée à s'engager). Le rapport entre identité personnelle et transaction est alors réflexif: transaction et identité personnelle se définissent mutuellement. La société est constituée de l'ensemble de ces transactions; par ailleurs, le jeu de négociation est contraint par le champ créé par le canevas des transactions déjà socialement reconnues et sanctionnées.

Quel que soit le champ culturel dans lequel on se trouve (boucher, boulanger, infirmière, ingénieur, soldat, etc.), l'identité sociale est déterminée par la nature des engagements qu'on s'est vu confier dans nos rôles d'agent, de patient et de bénéficiaire. Changer de champ c'est aussi changer d'identité. D'ailleurs, puisque la langue fournit un système de classification (Whorf, 1956) et que la classification repose sur une base élémentaire de contrastes semblables à ceux que nous avons décrits (Greimas, 1966, 1970 ; Taylor, 1978), il s'ensuit que l'organisation est à la fois sémantiquement et pragmatiquement structurée. Le bureau n'est pas seulement un lieu pour l'exécution des tâches; il est aussi l'accès à une identité sociale.

\section{Prémisse}

Il existe deux sortes d'acteur : les micro acteurs et les macro acteurs.

70 Notre théorie distingue la transaction (ce qui véhicule une relation) de l'interaction (la séquence de comportements verbaux et non-verbaux par le truchement de laquelle la transaction doit être réalisée). C'est la reconnaissance de la transaction dans l'interaction qui sous-tend la production de l'organisation dans la communication.

71 Cette première distinction en engendre une autre entre deux niveaux logiques d'intervenant, soit l'agent et l'acteur. Le concept d'agent relève du niveau de la transaction, celui d'acteur de l'interaction. La définition d'agent est dans le domaine du générique; elle fait partie du savoir collectif en ce qui a trait aux tâches à accomplir et aux relations à maintenir. Quant à l'acteur, il relève du domaine de l'individuel, du circonstanciel. L'acteur joue le rôle d'agent, au même titre que l'acteur sur scène qui entre dans la peau d'un personnage du caractère qu'il représente. C'est l'acteur qui entre dans la conversation; les engagements qu'il y entreprend le transforme en agent et lui confie son statut social, sa légitimité et son autorité. 
72 Mais le langage nous permet d'entrer dans une conversation de différentes manières. Un micro acteur ne parle strictement que pour lui-même. Quand il s'engage, c'est strictement en son propre nom, et non pas en celui d'un autre. Il, ou elle, parle comme un « je ».

Un macro acteur (Callon \& Latour, 1980) agit pour d'autres autant que pour lui-même. Il s'engage au nom d'une collectivité. Il s'approprie le terme «nous ». Il devient ou tente de devenir - l'intermédiaire par lequel une personne morale s'exprime. Ses intentions (et donc le sens de ses interventions), en bonne partie, sont celles de son groupe. Il s'annonce officiellement comme un agent par le choix même du pronom.

Par ailleurs, le macro acteur une fois reconnu fait plus que représenter son groupe. Il peut (et dans une certaine mesure, doit) synthétiser en lui (et, non pas seulement dans son expression) la volonté d'une association de gens -la manifestation extrême du collectif dont il dépend. Bien qu'autorisé à parler en leur nom, le rôle assumé ne se limite pas à celui de porte-parole ; la logique de la distinction transaction/interaction dicte qu'au plan de l'interaction l'agent se manifeste comme individu reconnaissable, et non seulement comme agent immanent. Lorsqu'il s'engage dans une conversation, et tente d'exécuter un acte de langage, c'est son intentionnalité qui doit être évaluée par les autres, puis confirmée ou non. Lorsque son acte est réussi, il a, par là même, engagé le collectif qu'il représente et son intentionnalité, exprimée et confirmée en interaction, se confond avec celle de son groupe.

L'identité de toute collectivité, comme de toute autre personne, est associée à un ensemble de transactions dans lequel elle s'engage. Mais, pour s'engager dans une conversation (et ainsi pour réaliser une transaction) il faut pouvoir parler d'une seule voix. Le macro acteur est cette voix. La « boîte noire » dont parle Callon \& Latour (1980) a été fermée, la structuration du pouvoir de l'organisation est ainsi confirmée. La capacité de l'individu à assumer le rôle de macroacteur (et donc de devenir agent de la collectivité) est un indice de son pouvoir.

Lorsque deux (ou plus de deux) macroacteurs interagissent, on est alors en présence d'une conversation strictement organisationnelle. La forme extérieure d'une telle conversation ne se distingue pas de toute autre conversation, car les mêmes règles d'interaction s'appliquent, mais la substance y est totalement différente.

\section{Prémisse}

\section{La conversation est le véhicule par le truchement duquel des transactions sont réalisées.}

Nous sommes maintenant prêts à répondre à la question que nous avons posée au début à savoir comment une transaction se réalise dans l'interaction.

Dans certaines situations - lorsque, par exemple, on achète un produit dans un magasin les règles d'interaction, et le caractère de la transaction propre à cette circonstance, sont si bien connus que la transaction peut se réaliser sans la moindre ambiguïté. Dans d'autres contextes, par ailleurs, il peut subsister une équivoque. La nature de la transaction apparaît seulement pendant le déroulement de l'interaction ou peut même devenir claire seulement après. L'interaction ne sert pas qu'à mettre la transaction en scène, mécaniquement (une performance), mais doit simultanément la construire.

Notre conception du processus de construction d'une transaction se base sur la théorie des actes de langage (« speech acts », en anglais) de John Austin (1960). Selon cette théorie, 
le langage n'est pas seulement un instrument ancillaire pour l'accomplissement des transactions, il est lui-même un médium transactionnel en soi : échanger des vœux dans une cérémonie de mariage réussit une transaction aussi réelle que d'échanger une maison pour une somme d'argent par un acte de vente. L'« objet de valeur » échangé n'est pas obligatoirement, ou même le plus souvent, un objet matériel, ou un service, mais peut être quelque chose d'aussi intangible qu'une information, un conseil, une commande, une critique, une autorisation, etc.. Des actes aussi habituels que des affirmations, des requêtes, des instructions, des promesses, des louanges ont tous le caractère d'un échange et entrent donc dans la définition d'une transaction. Toute conversation se prête potentiellement à la réalisation de transactions, et donc à l'affirmation de l'identité des parties concernées, ainsi que de leurs droits et obligations.

La théorie des actes de langage présume que les circonstances sont déjà connues (lieu, temps, identité des participants, nature de l'occasion sociale, etc.). On prend pour acquis que les deux interlocuteurs maîtrisent tous les deux un langage commun et qu'ils savent exécuter les étapes de l'interaction qui composent un échange verbal. Le thème de l'interaction (l'objet de l'échange) doit, par ailleurs, être établi dans l'échange. C'est l'initiateur de l'échange, présument motivé par une intentionnalité de produire un état de fait nouveau qui ne peut pas se réaliser sans la collaboration du destinataire, qui définit l'objet de l'échange. C'est le destinataire qui accepte ou non la définition de la situation ainsi offerte.

Quand toutes ces conditions sont satisfaites, y compris l'acceptation par la cible de l'interpellation initiale, il y a un acte de langage, au sens d'Austin. Mais il y a aussi une transaction : un objet de valeur, bien que revêtant une forme purement symbolique, ainsi qu'une source et une destination, ont été spécifiés et il y a eu un transfert de cette valeur.

Austin croit avoir identifié les mécanismes de la langue qui permettent cette réalisation d'un acte par le truchement de l'interaction. Les phrases d'une langue sont d'abord des locutions. En tant que locutions, elles peuvent (doivent, en effet) spécifier un thème ou un objet de référence (qui peut être concret ou abstrait) et un état de cet objet (c'est ainsi que l'objet de l'échange est fixé). Mais une phrase peut spécifier simultanément, sur une autre dimension, ce qu'Austin appelle une force illocutoire. En tant qu'illocution, le langage ne sert plus seulement en tant qu'instrument de description, mais instaure luimême un état. Supposons qu'une personne A interroge une personne B sur sa connaissance d'une situation particulière. Le contenu explicit de l'échange - la locution est l'information communiquée de B à A. C'est la matière de l'échange. Mais en prenant l'initiative d'ouvrir un échange et de formuler son intervention sous la forme d'une question $\mathrm{A}$ réclame la coopération de $\mathrm{B}$ et si ce dernier se plie à la demande, il s'est mis dans la position d'accepter ainsi l'autodéfinition de $\mathrm{A}$ comme celui qui mène. La force illocutoire de la question initiale est donc l'annonce (et non pas la description) d'un acte à venir qui prend la forme d'une collaboration. Selon notre terminologie, A est l'agent de l'échange et $B$ le bénéficiaire (ou la victime, suivant l'interprétation de l'échange). L'effet exercé sur B est nommé, par Austin " effet perlocutoire». Le langage fournit déjà des options permettant d'exprimer une force illocutoire: les modes déclaratif, interrogatif, impératif, etc., mais la force illocutoire s'exprime par tout un ensemble de moyens, y compris des indices paraverbaux et nonverbaux.

Le facteur déterminant dans l'interprétation d'une illocution par sa cible est la reconnaissance de l'intention de l'initiateur de l'échange. Prenons le cas d'une promesse, par exemple. Réaliser une promesse suppose que la source de l'acte prononce une 
formule telle que "Je vous promets...», que le destinataire reconnaît que c'est véritablement l'intention de la source de commettre l'action décrite à un temps futur et que le destinataire accepte le geste ainsi offert. Si seulement une de ces conditions n'est pas respectée, la promesse est invalidée et l'acte est raté.

De la même façon, une transaction peut ainsi avorter. La distinction entre acte et transaction est que cette dernière s'insère dans un système d'interactions : l'acte qu'elle comporte laisse une trace, soit l'obligation de récompenser le don (ou le vol) par un acte subséquent -une dimension qui est absente dans la théorie des actes de langage.

Ce qui rend l'accomplissement d'un acte de langage (ou d'une transaction) problématique est la possibilité d'une mauvaise interprétation de l'intention de la source par le destinataire. L'interprétation conditionne l'acceptabilité de l'acte offert. Parce que le sens d'un acte de langage n'est pas capté uniquement par une lecture du sens de la locution (sur le plan de la linguistique), mais implique aussi une attribution de l'intentionnalité sousjacente, les deux participants sont potentiellement engagés dans ce que Goffman (1957) a appelé un « jeu d'information » où tout comportement, non-verbal aussi bien que verbal, peut être lu pour des signes cachés de la vraie intention du locuteur. Une telle situation, où chacun des partenaires de l'échange essaie de suppléer à l'inadéquation possible de l'énoncé explicite par sa connaissance de la situation (et d'autres situations semblables jugées typiques), ainsi que par ses intuitions concernant la motivation de son partenaire à l'échange, est une instance de L'indexicalité. La locution énoncée n'est qu'un indice de la véritable signification de l'illocution.

Le caractère auto-organisateur de la conversation

89 Nous découvrons maintenant une propriété fondamentale de la conversation: la réflexivité.

Nous avons vu qu'une transaction est un acte qui comporte la transmission d'une valeur, matérielle ou symbolique: le destinateur est informé par la source. Cependant, la transaction est simultanément la production d'une valeur. Prenons le cas d'un groupe de travail qui s'est réuni pendant une après-midi à la suite duquel ils sortent collectivement un rapport qui contient des recommandations. Au cours de leurs discussions de multiples transactions ont eu lieu: les individus échangeaient leurs connaissances, leur suggestions, leurs avis, etc. Mais le rapport produit à la fin constitue une production qui les transforme de micro acteurs en macro acteurs et crée ainsi le préalable à une autre transaction future où le groupe dans son ensemble figure comme agent. La conversation est alors, à la fois, un moyen pour l'échange des valeurs et pour leur création (des données brutes transformées en information). Tout autre processus de production suppose l'éventuel épuisement des ressources naturelles subséquentes à leur conversion en produit culturel utilisable: le processus est irréversible. Dans la conversation, la "matière brute " est l'information, mais le "produit» est aussi l'information. La «ressource naturelle» n'est jamais épuisée, tout en étant constamment transformée. Cette propriété réflexive de la conversation explique le caractère auto-organisant de la communication et sa capacité de supporter indéfiniment l'organisation. 


\section{La réification (traduction en chose) de l'organisation par sa textualisation}

Une organisation, prise dans sa manifestation de la conversation, est un système d'interaction par le biais duquel des transactions sont réalisées. Mais à ce niveau l'organisation n'est pas réelle, elle est plutôt hyperréelle. Par hyperréelle nous voulons dire que l'organisation est une superposition de toutes les réalités de ces membres, pas nécessairement cohérentes les unes avec les autres, et même parfois en forte contradiction. Aucune ne jouit du statut de la réalité. Chacune est aussi valide que les autres. Pour qu'une seule organisation puisse naître il faut que cette construction intersubjective reçoive le statut d'objet. Cette transformation est effectuée par la réification du système transactionnel sous forme de texte. C'est grâce à cette transformation que se constitue l'organisation.

La narrativisation de la conversation

L'interaction se caractérise comme une séquence continue d'échanges verbaux, sans coutures naturelles autre que le principe d'alternance des locutions (Watzlawick et at, 1967, p. 54). Une transaction a, par définition, un début, un développement et une fin : on doit l'ouvrir et la conclure. Pour détecter la transaction dans l'interaction il faut donc insérer des parenthèses (créant de cette façon des ouvertures et des fermetures). II faut, autrement dit, « ponctuer » la séquence d'interactions (Watzlawick et al, 1967, p. 54) afin de lui donner une forme qui correspond à celle des actes de langage, les véhicules de la transaction.

Or, une séquence d'actions où il $\mathrm{y}$ a un début et une fin, et où il $\mathrm{y}$ a eu un développement qui correspond à un échange de valeur entre le début et la fin (une inversion), correspond aussi à la définition d'une histoire simple ou épisode narratif (Prince, 1973). La forme d'une transaction est isomorphe à celle d'un épisode dans une histoire. Les interlocuteurs de l'interaction peuvent être conçus alors, dans la perspective narrative, comme étant des protagonistes dans une histoire (Mandler \& Johnson, 1977) où l'échange est un épisode.

Une analogie utile est celle du « cadrage ». Tout artiste sait que la scène devant lui cache une infinité de peintures potentielles; c'est en encadrant certains des composantes de la scène (et ce faisant, en les sélectionnant et en excluant d'autres) que l'œuvre d'art est créée. De façon semblable, c'est par l'acte d'encadrement que l'interaction est transformée en unité transactionnelle et que, par conséquent, l'organisation peut émerger du bruit ambiant (Saumier et Taylor, 1990). Le cadrage transforme ainsi l'interaction en scène ou en situation et ce faisant permet son interprétation et son appréhension. La situation est composée d'un lieu, d'un temps et d'un ensemble de personnages, c'est-à-dire tout ce qui est typiquement circonstanciel.

La narrativisation de la conversation constitue la première étape de sa textualisation.

Cette transformation est appelée par Ricoeur (1986) une distanciation.

Selon lui, la modalité discursive de la conversation est réalisée selon le mode du temporel et se déroule dans le présent. Tout système de langage (dont la narration dépend) est, par contraste, virtuel et indépendant du temps. Dans le contexte de la conversation, le sujet (celui qui parle) et l'objet indirect (celui qui écoute) sont nécessairement présents et identifiables : l'« instance de discours » (un terme emprunté par Ricœur à Benveniste) est rendue autoréférentielle par des indices linguistiques tels que des pronoms. Le langage 
n'a pas de sujet dans le même sens car le dit est séparable du dire. Finalement, la conversation concerne toujours un état de fait dans un monde réel, une situation qu'elle tente de décrire, d'exprimer ou de représenter. Le langage constitue, par contraste, le code qui permet l'expression mais en soi il est composé de signes qui se réfèrent mutuellement. Il ne se réfère à aucun monde. Le discours est réalisé en tant qu'événement (ce que nous avons appelé « interaction ») mais il est compris comme ayant un sens. Ce que nous voulons comprendre, comme participants à l'interaction, ce n'est pas l'événement fugitif mais le sens qui persiste. Ici Ricoeur, tout comme nous, fait appel à la théorie des actes de langage : le sens de l'interaction apparait dans sa compréhension comme un ensemble d'actes médiatisés par l'interaction. Mais les actes font aussi partie de l'univers de la narration. C'est ainsi que l'interaction est extériorisée et son inscription en texte est rendue possible. Le social et le cognitif (ou ce que Halliday, 1970, appelle l'interpersonnel et l'idéationnel) se rejoignent dans ce même acte. L'interaction suit la règle du syntaxique, la transaction se conforme à celle du sémantique. La signification du geste interactif provient du fait qu'on peut le lire comme un élément dans une histoire. C'est ainsi qu'on décode le sens de l'interaction. Tout compte-rendu verbal de l'interaction est une traduction de la conversation en texte virtuel. La narrativisation précède la textualisation. L'inscription du compte-rendu produit un texte matériel.

Le texte ainsi produit est associé initialement avec un auteur, dans un contexte spécifique de production mais c'est le propre du texte de pouvoir se détacher de ses circonstances particulières de création et de devenir un objet en soi - une œuvre ou un artefact. Le texte organisationnel diffère cependant de l'œuvre de fiction (sur laquelle Ricoeur centre son attention) dans sa pré tension de décrire un monde réel (et non pas purement imaginaire) et même de servir de modèle et de guide pour la conduite des transactions dans le système d'interaction qu'il décrit. Ce modèle d'action est composé de scénarios(ce que Schank \& Abelson 1977 appellent des scripts). Un scénario est un ensemble d'instructions dont le but est de guider les comportements d'un acteur dans une situation donnée - sa fonction est générique. Le scénario est formulé dans des termes généraux, laissant nécessairement beaucoup à la discrétion de l'acteur. Les « acteurs » sont en effet des agents, ou acteurs typiques (macro-acteurs) : médecins, avocats, banquiers, patrons, secrétaires, ainsi de suite. Chaque profession est caractérisée par ses propres scénarios.

Le script fournit un plan explicatif pour entrer dans l'interaction. Il s'ensuit que la conversation est médiatisée par les textes, mais il est également vrai que les textes sont médiatisés par la conversation, car le scénario, étant énoncé seulement en généralités, doit être réalisé dans l'interaction. La conformité de la réalité interactionnelle au scénario imaginaire n'est jamais qu'approximative. Par ailleurs, l'interprétation de la conversation par le texte ne peut jamais être que partielle étant donné que dans le passage du spécifique conversationnel au général textuel il y a obligatoirement une perte de variété et une équivoque résiduelle.

\section{Entre la conversation et le texte}

102 C'est ici que nous confrontons le paradoxe de l'organisation humaine et son principe de changement. 
La réalisation d'une transaction par le biais d'une interaction suppose une narrativisation simultanée par les participants car c'est par la transformation en histoire que la transaction acquiert son sens. Or, nous avons constaté une asymétrie fondamentale : les perspectives du patient et de l'opérateur, et de l'agent et du bénéficiaire, ne peuvent pas coïncider entièrement car leur investissement dans l'objet de valeur est différent. Mais le texte organisationnel n'est pas multidimentionnel : parce qu'il appartient à la sphère du narratif il exprime nécessairement un point de vue (et en supprime un autre). Passer du mode de la conversation à celui du texte constitue une perte de variété.

Le cas est inversé dans le passage du texte à la conversation. Une transaction ne peut être réalisée que par un effort de collaboration. L'interactivité suppose une alternance - un zigzag - de comportements individuels séquentiellement, et générés en collaboration avec les personnes en présence. Ces interventions de particuliers peuvent se combiner afin de produire une transaction, mais elles peuvent aussi la rater. L'interactivité ne garantit pas la «transitivité». L'accomplissement de l'interaction suppose bien sûr une compétence - une maitrise des règles de la grammaire - de la part des deux partenaires, d'abord afin de produire les phrases de la langue (compétence linguistique), et ensuite afin de gérer la séquestration permettant de produire des suites cohérentes d'interaction (compétence interactionnelle). Mais achever une transaction n'est pas seulement une question de compétence (communicationnelle ou autre). Bien que la compétence communicationnelle se doivent d'être partagée par les deux partenaires afin que puisse s'achever l'échange et s'instaurer la relation il y a plus : il faut l'acceptation ou le rejet de l'échange de valeur ainsi communiqué. Puisqu'une transaction suppose un gain ou une perte de valeurs, elle laisse des traces, sous forme d'une dette ou d'une obligation à acquitter, ou une rétribution à effectuer ou à subir, a une date ultérieure. Accepter une transaction, c'est s'engager moralement. Bien que la conversation soit le médium de l'organisation toute conversation ne réussit pas en tout temps la production d'organisation par la réalisation des transactions. Les transactions, souvent, doivent être négociées.

Entre le texte et la conversation il y a donc un gain de variété.

C'est cette incompatibilité de modes de communication qui explique la dynamique de changement organisationnel: le texte (en l'absence duquel il n'y a aucun objet d'organisation mais seulement des cultures de conversations) ne représente qu'imparfaitement la conversation et la conversation dépasse constamment les limites fixées par le texte.

\section{Le changement}

Le changement peut provenir de deux sources : la conversation ou le texte.

Le changement qui émane du texte

Un changement qui commence par une innovation textuelle, dans une conversation de macroacteurs, et qui est subséquemment imposé par fait sur la conversation organisationnelle, s'appelle déductif ou « top-down ».

Ce genre de "changement" est très répandu dans notre société même s'il ne réussit presque jamais à atteindre ses objectifs. Sa popularité s'explique par l'illusion de pouvoir que la formulation d'un texte donne aux instances décisionnelles composées de macroacteurs. Son manque de succès s'explique par un phénomène peu investigué dans la 
littérature, soi l'auto gérance de la conversation par les acteurs qui la soutiennent par leur pratique transactionnelle.

\section{L'auto-gérance de la conversation}

111 Une conversation soutenue par une communauté de membres stables est extrêmement élastique, lorsque confrontée à une tentative de changement venant de l'extérieur. La stabilité est assurée par un principe d'auto-régulation où le texte généré au sein du groupe sert de stabilisateur (Cooren inédit). Le texte fournit au groupe un moyen d'interprétation de ses actions et de leurs significations. Cette signification inclut une attribution des identités des acteurs dans le groupe et donne ainsi un sens à leur vie. Le texte, à son tour, est constamment renforcé par la conversation, qui lui confère une légitimité certaine, par le fait même d'être respectivement interpellé. Tout texte importé dans un groupe, quelle que soit son autorité apparente, n'est pas simplement rejeté (il peut même être superficiellement accueilli); il n'est même pas compris à moins d'être en conformité avec le texte existant. Le résultat de la plupart des campagnes visant à transformer la culture, ou la pratique, ou la structure des activités d'une organisation à composition stable est donc une acceptation officielle du nouveau régime accompagnée par une absence de vrai changement.

\section{Le changement généré par la conversation}

Un changement qui commence par une évolution de la conversation et qui amène à un ajustement du texte s'appelle inductif ou "bottom-up». Cet ordre de changement organisationnel rencontre une autre difficulté, soit l'élasticité du macro texte de l'organisation.

113 Une très grande partie des transactions d'une organisation sont répétitives, soit par la manière qu'on traite des interlocuteurs extérieurs (clients ou fournisseurs) soit par l'adoption de pratiques de gestion normalisées (pour ce qui est de l'embauche, la promotion, l'administration des dossiers, la prise de décision, etc.). Mais toutes les relations ne sont pas déjà déterminées à l'avance. Le "couplage " d'une organisation (Weick, 1979) demeure suffisamment lâche pour qu'il y ait toujours un espace pour des tractations qui exigent un certain entrepreneur ship. On parle ici de toute la partie de la vie organisationnelle qui implique des jeux politiques. De ces transactions non-prévisibles émergent de nouvelles formes d'association et une évolution progressive de l'organisation. C'est la principale source de variété et de nouveauté dans l'organisation. De ce commerce transactionnel improvisé émergent aussi de nouveaux textes.

114 Mais ces nouveaux textes ne sont pas ceux de l'organisation dans son ensemble. Chaque organisation se dote d'une définition d'elle-même - de son rôle, de sa mission, de son mandat, de sa culture, de ses valeurs. Tout nouveau texte constitue une forme d'hérésie. $\mathrm{Si}$, comme nous l'avons vu, la conversation des groupes dans une organisation est supportée par leur texte, il est aussi vrai que le texte de l'organisation est fortement supporté par la macro conversation, aussi fortement que l'est le texte orthodoxe de la communauté scientifique lorsqu'elle est confrontée à une menace de changement de paradigme (Kuhn, 1970). 


\section{Le principe de l'abduction}

115 Un changement qui aboutit à une modification interactive du texte et de la conversation s'appelle abductif. Comme nous l'avons vu, l'organisation peut introduire de nouveaux textes sans que la conversation évolue véritablement. De façon similaire, la conversation peut prendre de nouvelles formes sans que ces modifications se trouvent reflétées dans le texte officiel de l'organisation. Dans chacun de ces cas, il y a peut-être l'illusion d'évolution mais c'est une illusion jusqu'à ce qu'il y ait un changement dans l'articulation des deux modes.

Comment le changement se réalise-t-il dans un esprit d'abduction?

L'organisation et l'environnement dans lequel elle évolue sont constamment en transition d'une réalité à une autre. Il n'est pas nécessaire d'engendrer le changement - il est omniprésent parce que la variété de la conversation est toujours plus grande que celle du texte. La tâche de la direction n'est pas de déterminer le sens du changement car ce dernier est hors le contrôle de tout acteur aussi puissant soit-il. Le rôle du gestionnaire n'est pas de «diriger » mais de comprendre et de formuler un texte qui reflète plus fidèlement que l'ancien la nouvelle situation. Il ne peut pas diriger (une idée désuète) mais il peut, le cas échéant, orienter la conversation en réunissant de nouvelles manières de l'interpréter et en lui donnant un nouveau sens ainsi en créant une nouvelle narration. Cette tâche suppose une vision nouvelle des capacités requises du leader, qui le rapproche $\mathrm{du}$ profil du scientifique, c'est-à-dire quelqu'un capable de traduire le dossier de transactions en évolution en cadre explicatif adéquat et capable de rallier les membre de l'organisation autour de cette nouvelle interprétation. Il s'agit de ce que nous appelons l'« hypergestion » (Taylor \& Van Every 1993).

\section{Le contexte actuel}

118 Les transformations technologiques des années récentes, en télé communication, en informatique et en transport à distance, ont créé une population de travailleurs beaucoup plus mobile qu'auparavant. Le résultat a été de stimuler l'émergence accélérée de nouvelles formes d'association et de nouveaux modèles transactionnels. Les textes organisationnels dont nous avons hérité n'ont pas évolué au même rythme. Il y a maintenant un fossé important entre la réalité organisationnelle contemporaine et notre idée de l'organisation. Les "anomalies» (pour utiliser un terme rendu populaire par Kuhn, 1970) se sont accumulées. Une opportunité unique s'ouvre aux étudiants de la communication de répondre à ce qui est devenu une crise épistémologique dans les sociétés de la modernité. Ceci nous paraît être le véritable défi du chercheur contemporain en communication. 


\section{BIBLIOGRAPHIE}

\section{RÉFÉRENCES}

Akzam, H., Implantation technologique et adaptation organisationnelle: Période d'expérimentation. Mémoire de maîtrise, Département de Communication, Université de Montréal 1991.

Austin, J., How to do things with words. Harvard University Press, Cambridge, Mass. 1962.

Bateson, G., Steps to an ecology of mind. Ballantyne, New York 1972.

Callon, M., \& Latour, B., «Unscrewing the big Leviathan : How actors macro-structure reality and how sociologists help them to do so " dans A. Cicourel, \& C. Knorr-Cetina, Advances in social theory and methodology : 277-383, 1981.

Chandler, A. D., Jr., The visible hand : The managerial révolution in American business. Harvard University Press, Cambridge, Mass. 1977.

Clemons, E. K., \& M. C. Row, Information technology and économie reorganization. Wharton School of Business, University of Pennsylvania, 1989.

Cooren, F., Le modèle de l'auto-organisation : application à l'analyse du discours. Actes du 4 ième Colloque sur la communication organisationnelle, Université de Montréal, le 12 novembre 1992.

Dewey,J., Democracy and éducation Free Press, 1944 ed. : Macmillan, New York 1916.

Garfinkel, H., Studies in ethnomethodology. Prentice-Hall, Englewood Cliffs, N.J. 1967.

Giroux, N. \& J.R. Taylor, (accepté) A conversation/text model of organizational communication. Colloque de l'EGOS « The production and diffusion of managerial and organisational knowledge ». École Supérieure de Commerce de Paris, 6-8 juillet, 1993.

Goffman, E., The présentation of self in everyday life. Doubleday, New York 1959.

Greimas, A.J., Sémantique structurale. Larousse, Paris 1966.

Greimas, A.J., Du sens. Le Seuil, Paris 1970.

Gurd, G. (en préparation). An interprétative approach to studying organizational culture concerning computerization in a hospital: Tasks and couplings. Thèse de doctorat, Sciences de la communication, Université de Montréal.

Halliday, M. A. K., Language structure and language function. dans J. Lyons, New Horizons in Linguistics : 140-165, Penguin, Harmondsworth, Middlesex, England 1970.

Hovey, M., Conversation in the software development process. Master's thesis, Concordia University, Montréal 1992.

Jourdenais, M., Changements organisationnels et reconstruction de marges de manoeuvre à la Société des Postes. Mémoire de maîtrise, Département de communication, Université de Montréal, 1992.

Kondo, D. K., Crafting selves : Power, gender, and discourses of identity in a Japanese workplace. The

University of Chicago Press, Chicago 1990. 
Kahn, T. S., The structure of scientific révolutions (2ième éd.), The University of Chicago Press, Chicago 1962, 1

Lemer, L. (en préparation). A discourse analysis of the Governance in an information society roundtable process. Thèse de doctorat, Sciences de la communication, Université de Montréal.

LeShan, L. \& H. Margenau., Einstein's space \& Van Gogh's sky : Physical reality and beyond. Collier, New York 1982.

Mandler, J. M., \& N. S. Johnson, Remembrance of things parsed : Story structure and recall. Cognitive Psychology, $9: 111-151,1977$.

March, J. G., \& H. A. Simon, Organizations. John Wiley, New York 1958.

Mauss, M., Essai sur le don : Forme et raison de l'échange dans les sociétés archaïques. Année sociologique, seconde série, $1: 1923-4$.

Mead, G. H., Mind, self and society. University of Chicago Press, Chicago 1934.

Mintzberg, H., The Nature of Managerial Work. Harper \& Row, New York 1973.

Mintzberg, H., Structure et dynamique des organisations. Trad. de The structuring of organizations. Les Éditions d'organisation, Les Éditions d'ARC, Paris/Montréal 1979/1982.

Morgan, G., Images de l'organisation. Les Presses de l'Université Laval, Éditions ESKA, Québec 1986/1989.

Porter, M. E., Competitive strategy. The Free Press, New York 1980.

Porter, M. E., Competitive advantage. The Free Press, New York 1985.

Prince, G., A Grammar of stories. The Hague : Mouton, 1973.

Prince, G., Narratology : The form and functioning of narrative. The Hague : Mouton, 1982.

Ricoeur, P. Qu'est-ce qu'un texte ? expliquer et comprendre. (Édit. D. Rasmussen) Mythic-symbolic language and philosophical anthropology. Martinus Nijhoff, La Haie, 135050,1971.

Ricoeur, P. La fonction herméneutique de la distanciation, dans F Bovon \& G. Rouiller, Exegesis : Problèmes de méthode et exercices de lecture. Delachaux et Niestlé, Neuchâtel : 201-215,1975.

Ricoeur, P., Du texte à l'action : Essais d'herméneutique, II, Le Seuil, Paris 1986.

Robichaud, D., Une analyse du concept d'enaction. Mémoire de maîtrise, Sciences de la communication, Université de Montréal 1991.

Salancik, G. R. \& H. Leblebici, Variety and form in organizing transactions : A generative grammar of organization. Research in the Sociology of Organizations (JAI Press), $6: 1-31,1988$.

Saumier, A. (en préparation). L'implantation d'un nouveau programme de Sciences humains au collégial : une analyse macro-conversationnelle de l'action institutionnelle. Thèse de doctorat, Sciences de la communication. Université de Montréal.

Saumier, A. \& J.R. Taylor, Organizational conversation : Interprétation, interaction and commitment. Paper presented at the 40th Conférence of the International Communication Association, 27 mai, Dublin 1990.

Schank, R., \& R. Abelson, Scripts, plans, goals and understanding. Lawrence Erlbaum, Hillsdale, N.J. 1977.

Searle, J., Speech acts : An essay in the philosophy of language. Cambridge University Press, Cambridge, England 1969. 
Searle, J. R., A classification of illocutionary acts. Language in Society, 5 :1-23,1976.

Searle, J. R., Intentionality : An essay in the philosophy of mind. Cambridge University Press, New York 1983.

Silverstone, R., E. Hirsch \& D. Morley, Information and communication technologies and the moral Economy of the household. Dublin, Ireland 1990.

Taylor, J. R., Télévision crime drama : A mythological interprétation. Report of the Royal Commission on Violence in the Communications Industry, vol. 3 Violence in télévision films and news : 157-219,1976.

Taylor, J. R., A method for the recording of data and analysis of structure in task groups. Doctoral Dissertation, University of Pennsylvania. University Microfilms, Ann Arbor, Philadelphia, USA 1978a.

Taylor, J. R., Modeling the task group as a partially self-programming communication net : A cybernetic approach to the study of social processes at the small group level. dans K. Krippendorff, Communication and control in society, Gordon \& Breach, New York 1978b.

Taylor, J. R., Steps to a generative grammar of the office. Collected papers on the psychological and social effects of vidéotex, Ministère des communications, Ottawa 1981.

Taylor, J. R., Computer aided message Systems : An organizational perspective, dans N. Naffah, Office information Systems : 631-651. INRIA/North Holland PublishingCo,1982.

Taylor, J. R., Conceptual impediments to productivity. Optimum, a forum for management, $141 / 2: 19-42,1983$.

Taylor, J. R. New communication technologies and the émergence of distributed organizations : Looking beyond 1984, dans L. Thayer, Organization Communication : Emerging perspectives, Ablex, Norwood, N.J. 1986.

Taylor, J. R., Une organisation n'est qu'un tissu de communications. Cahier de recherche en communication, Département de Communication, Université de Montréal, Montréal 1988a.

Taylor, J. R., Communication theory: Can it explain the organizational « paradigm révolution ». Rapport interne du Groupe d'intervention en communication organisationnelle (GISCOR), Département de Communication, Université de Montréal 1988b.

Taylor, J. R., Agents, patients and beneficiairies : How organizational reality gets constructed. Communication, Congrès annuel de l'International Communication Association, New Orléans, Louisiana, le 31 mai 1989.

Taylor, J. R., Une théorie tridimensionnelle de la communication organisationnelle. Communication/ Information, $111: 17-59,1990$ a.

Taylor, J. R., Trans cultural models of organizational communication : A Canadian perspective. Communication, Congrès annuel de l'International Communication Association, Dublin, Ireland, le 26 juin $1990 b$.

Taylor, J. R., Rethinking the theory of organizational communication. Ablex, Norwood, N.J. 1993.

Taylor, J. R, Worldview as a key to the effectiveness of computer-supported communication Systems..

« Invitational workshop » sur « Social science research, technical Systems and coopérative work », CNRS, Quai Anatole France, 8-11 mars, Paris 1993.

Taylor, J. R. \& N. Giroux, Organizational meaning through communication : A text-oriented approach. 
Communication, le 3ième Conférence on Organizational Symbolism and Corporate Culture (sCos), Milan, Italie, le 24 juin 1986.

Taylor, J. R. \& E. J. Van Every, The vulnérable fortress : Bureaucratie organization and management in the information society. University of Toronto Press, Toronto 1993.

Taylor, J. R. \& D. Robichaud, A new look at enactment Conférence, Congrès annuel de l'International Communication Association, 22 mai, Miami, Floride 1990.

Watzlawick, P., J.H. Beavin \& D.D. Jackson, Une logique de la communication. Traduction de The pragmatics of communication. Éditions du Seuil, Paris 1967/1972.

Weick, K. The social psychology of organizing. Random House, New York 1979.

Weinshall, T. D., Managerial communication : Concepts, approaches and techniques. Académie Press, New York 1979.

Whitehead, A. N., Science and the modem world. Macmillan, London, New York 1925.

Whorf, B. L., Language, thought and reality : Selected writings of Benjamin Lee Whorf. MIT Press, Cambridge, Mass. 1956.

Williamson, O. E., Markets and hiérarchies : Analysis and anti-trust implications. The Free Press, New York 1975.

Williamson, O. E., The rise of modem business enterprise : Implications for industrial organization. University of Pennsylvania, 1977.

\section{RÉSUMÉS}

La plupart des théories de la communication prennent le concept d'« organisation » comme un donné. Dans l'article qui suit, nous proposons une approche différente, selon laquelle l'organisation devient une réalité par son émergence dans la conversation. Pour être comprise comme une organisation, la conversation doit être analysée comme un ensemble de transactions, permettant le tissage des relations, l'échange de valeurs et la constitution des identités. L'événement de la communication est analysé comme un lien complémentaire entre un agent et un bénéficiaire (victime). Pour que la transaction s'identifie dans l'interaction, il est nécessaire de narrativiser cette dernière. La narrativisation amène à la textualisation. C'est en tant que texte que l'organisation devient objet identifiable. La dynamique de changement se trace dans une dialectique entre la conversation et le texte.

Most theories of communication take the idea of « organization » as a given. The article which follows argues for a different approach, that the organization is a reality which emerges out of interaction. Organization becomes a reality when the conversation is understood as a set of transactions, by means of which relations are established, value is exchanged and identities are created. The nature of the communication event supporting transactions is analyzed as a linking of agent/beneficiary (victim) in a complementary relationship. Fundamental to the identification of a transaction is the narrativization of the conversation, preliminary to its textualization. It is as text that the organization becomes an identifiable object. The dynamics of change are traced to the ongoing dialectic of conversation and text. 


\section{AUTEUR}

\section{JAMES R. TAYLOR}

Université de Montréal

Le Professeur Taylor a été plusieurs fois appelé « le père de la communication organisationnelle au Canada français ». Il a été l'un des premiers canadiens a s'intéresser à cet aspect particulier de la vie des organisations. L'aspect le plus original de toute cette recherche, est sans aucun doute le développement d'une théorie communicationnelle de l'organisation. Elle est ni plus ni moins que l'aboutissement de toutes ses années de recherche dans ce domaine d'étude récent. 